\title{
Management of febrile neutropenia in pediatric cancer patients
}

\begin{abstract}
Introduction: Febrile neutropenia $(\mathrm{FN})$ is the most common complication that threatens the life of patients undergoing chemotherapy. It is considered an oncological emergency and its therapeutic decisions must be timely and accurate.

Objective: To evaluate the therapeutic approach given to pediatric cancer patients with episodes of neutropenia and fever.

Material and methods: Descriptive, observational, cross-sectional and retrospective study in children with acute lymphoblastic leukemia (ALL), acute myeloblastic leukemia (AML), Burkitt's lymphoma (BL) and lymphoblastic lymphoma (LL) who attended the hematology service from 2014 to 2018. Age, gender, underlying diagnosis, treatment phase, absolute neutrophil count, clinical manifestations, blood culture collection and results, initial antimicrobial therapy, treatment changes, complications, days of hospital stay and mortality were analyzed. Descriptive statistics was used, with measures of central tendency, percentages and frequencies with the SPSS v22 program.
\end{abstract}

Results: We identified 104 episodes of $\mathrm{FN}$ in 70 patients with cancer. The mean age was 7 years and majority of patients were male $(n=49,70.0 \%)$. ALL was the most frequent underlying malignancy $(\mathrm{n}=59,84.3 \%)$ and the highest amount of FN episodes occurred during the induction phase $(n=74,71.2 \%)$. Fever of unknown origin was observed in 29 (27.9\%) episodes. Blood cultures were collected in $14(13.5 \%)$ cases. The most commonly isolated microorganisms in blood culture were Gram-positive bacteria $(n=10,76.9 \%)$. $\beta$-lactams and aminoglycosides were the most common antibiotics used.

Conclusions: Febrile neutropenia is a frequent complication in the pediatric population with hemato-oncological diseases in our hospital. The therapeutic approach that patients receive is adequate.

Keywords: febrile neutropenia; leukemia; lymphoma; pediatrics
Volume 9 Issue I - 2019

\author{
Rey Manuel Hurtado Llamas,' María Elena \\ Haro Acosta, ${ }^{2}$ Joan Dautt Silva ${ }^{3}$ \\ 'Pediatric Hematologist, Gynecology-Pediatrics and Family \\ Medicine Hospital No. 31 of the Mexican Institute of Social \\ Security (IMSS). Mexico \\ ${ }^{2}$ D.C. and Pediatrician, Coordinator of Health Research, Baja \\ California Delegation of IMSS, México \\ ${ }^{3}$ Medical intern, Gynecology-Pediatrics and Family Medicine \\ Hospital No. 3 I of IMSS, Mexico
}

Correspondence: María Elena Haro Acosta, D.C. and Pediatrician, Coordinator of Health Research, Baja California Delegation of IMSS, Calzada Cuauhtémoc No.300, Colonia Aviación, 21230, Mexicali, B.C. México,

Email maria.haroa@imss.gob.mx,draeharo@gmail.com

Received: December 26, 2018 | Published: January II, 2019

\section{Introduction}

Febrile Neutropenia (FN) is defined as an absolute neutrophil count (ANC) $<500$ cells/microliter or $<1,000$ cells/microliter with a projected nadir of $\leq 500$ cells/microliter in the next 24 hours and fever $>101^{\circ} \mathrm{F}\left(>38.5^{\circ} \mathrm{C}\right)$ or two measurements $>100.4^{\circ} \mathrm{F}\left(>38.0^{\circ} \mathrm{C}\right)$ in one hour. ${ }^{1,2}$

Febrile neutropenia is the most common complication that threatens the lives of patients undergoing chemotherapy. In the United States, it is estimated that a total of 60,294 episodes of NF per year were recorded. ${ }^{3}$

In the context of oncological patients, neutropenia is more commonly associated with cytotoxic therapy. ${ }^{4}$

Bacterial infections are commonly caused by six main etiologies: three Gram-negative (E. coli, Klebsiella sp and Pseudomonas aeruginosa) and three Gram-positive (S. aureus, S. epidermidis and S. viridans). ${ }^{5}$

In order to give an adequate approach, a complete medical history and physical examination should be performed, as well as a complete blood count, a blood culture (taken before the start of antibiotic therapy) and a risk category should be established. ${ }^{6}$

According to the guidelines of the National Comprehensive Cancer Network (NCCN), patients who develop NF can be classified as low or high risk of complications. This classification takes into account comorbidities, functional status, temperature, source of infection, organ failure, etc. ${ }^{7}$

The 2017 update to the "Guideline for the Management of Fever and Neutropenia in Children with Cancer and Hematopoietic StemCell Transplantation Recipients", published in the Journal of Clinical Oncology ${ }^{8}$ recommends empirical therapy according to the patient's risk as follows:

- High risk; use monotherapy with an antipseudomonal $\beta$-lactam, a fourth-generation cephalosporin, or a carbapenem. In unstable patients, a second agent against Gram-negative bacteria or a glycopeptide can be added.

- Low risk: Consider initial or step-down outpatient management if infrastructure allows careful monitoring and follow-up

The Infectious Diseases of America (IDSA) guideline establishes that patients with febrile neutropenia must receive their initial doses of empirical antibacterial therapy within an hour after triage. ${ }^{9}$

Regarding hospital stay, it has been established that, during the first episode of FN, patients will be 1-10 days under surveillance and receiving intrahospitalary care. However, time usually increases with later episodes. ${ }^{10}$

The mortality rate in recent studies of febrile neutropenia in pediatric patients has ranged between $0.7 \%$ and $3.9 \% .{ }^{11}$ 
Repeated episodes lead to multiple sequelae such as infections by resistant microorganisms, prolonged hospital stays and decreased quality of life. They also constitute an important cause of mortality, so their detection and timely management have been considered a challenge. Therefore, the objective of this study was to evaluate the therapeutic approach given to pediatric cancer patients with episodes of neutropenia and fever in our hospital.

\section{Material and methods}

The present work is an analytical, observational, transversal and retrospective study of pediatric patients with cancer and febrile neutropenia treated at the Gynecology-Pediatrics and Family Medicine Hospital (GP-FMH) No. 31 of the Mexican Institute of Social Security (IMSS) from July 2014 to August 2018.

A thorough search in the registry of clinical files was carried out, locating patients with diagnosis of acute lymphoblastic leukemia (ICD-10 C910), acute myeloid leukemia (ICD-10 C92.0), lymphocytic lymphoma (ICD-10 C835) and Burkitt's lymphoma (ICD-10 C837).

We reviewed and identified those cases in which the diagnosis of febrile neutropenia was confirmed and included in our analysis all those that were aged 0 to 16 years. Files that had incomplete information were excluded.

We collected information on the following variables: age, gender, underlying diagnosis, treatment phase, absolute neutrophil count, clinical manifestations, nosological diagnosis, blood culture collection, microbial isolates, initial antimicrobial therapy, treatment changes, complications, days of hospital stay and mortality.

This study was conducted in accordance with the ethical principles of the Helsinki Declaration and our institution's Local Research Committee.

Descriptive statistics were used: measures of central tendency for quantitative variables and frequencies and percentages for qualitative variables; the information collected was analyzed in the statistical program SPSS version 22.

\section{Results}

Our search allowed us to identify 61 cases with ALL diagnosis, nine patients with AML, three with LL, and one with BL. After the application of inclusion and exclusion criteria, two patients with ALL were eliminated, one with AML and the LB case. Therefore, we analyzed 70 files in total.

During the study period, $104 \mathrm{FN}$ events were recorded. Of the total of patients included, 49 were men $(70.0 \%)$ and 21 women $(30.0 \%)$. The median age of the patients was 7 years (range of 2 to 16 years). The age medians by diagnosis and other general characteristics can be found in Table 1.

The average number of events that each patient presented was 1.5 (range 1-6). Majority ( $\mathrm{n}=74,71.1 \%$ ) of episodes occurred during the induction phase. The frequency of events according to treatment phase and diagnosis are summarized in Table 2.

Regarding the type of symptoms, the most common manifestations were respiratory $(\mathrm{n}=22,21.15 \%)$, digestive $(\mathrm{n}=21,19.8 \%)$ and dermatological $(\mathrm{n}=15,14.4 \%)$. In four $(3.8 \%)$ episodes, patients had mixed symptoms. In $13(12.5 \%)$ cases, they presented fever without symptoms. The frequency of each type of clinical manifestations is recorded in Figure 1.

Table I General characteristics according to diagnosis

\begin{tabular}{|c|c|c|c|}
\hline Characteristic & ALL & AML & $\mathbf{L L}$ \\
\hline Patients & 59 & 8 & 3 \\
\hline Male * & $45(76.3)$ & $3(37.5)$ & $1(33.3)$ \\
\hline Female * & $14(23.7)$ & $5(62.5)$ & $2(66.7)$ \\
\hline Age (years) ${ }^{+}$ & 7 & 5.5 & 4.5 \\
\hline FN episodes * & $93(87.7)$ & $8(8.5)$ & $3(3.8)$ \\
\hline Most common symptoms & Respiratory & Digestive & Digestive \\
\hline Absolute neutrophil count ${ }^{+}$ & $199 \times 10^{3}$ & $254 \times 10^{3}$ & $158 \times 10^{3}$ \\
\hline Deaths & $9(15.3)$ & $2(25)$ & $1(33.3)$ \\
\hline
\end{tabular}

* Number (percentage), + median, ALL, acute lymphoblastic leukemia; AML, Acute Myeloblastic Leukemia; LL, lymphoblastic lymphoma

Table 2 Treatment phases

\begin{tabular}{llll}
\hline Treatment phase & ALL $^{*}$ & AML $^{*}$ & LL $^{*}$ \\
\hline Induction & $66(71.0)$ & $6(75.0)$ & $2(66.7)$ \\
Consolidation & $9(9.7)$ & $2(25.0)$ & $1(33.3)$ \\
Maintenance & $7(7.5)$ & 0 & 0 \\
Relapse & $11(11.8)$ & 0 & 0 \\
Total & $93(100.0)$ & $8(100.0)$ & $3(100.0)$
\end{tabular}

*Number (percentage), ALL, acute lymphoblastic leukemia; AML, Acute Myeloblastic Leukemia; LL, lymphoblastic lymphoma

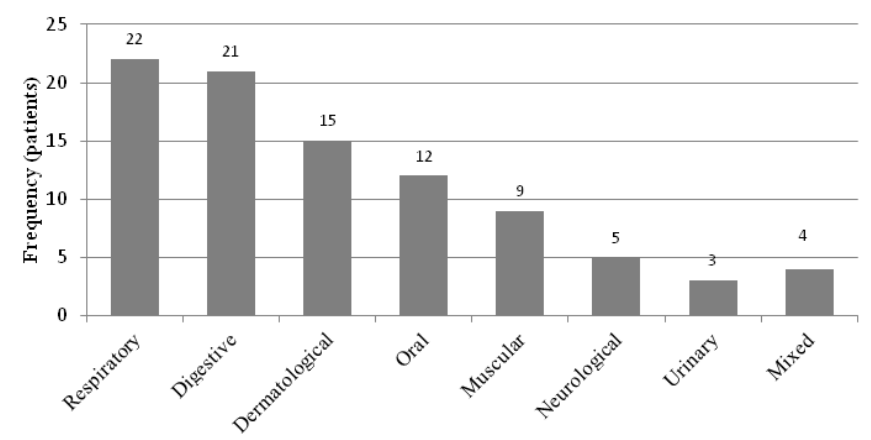

Figure I Frequency of clinical manifestations

The nosological diagnoses were: $29(27.9 \%)$ cases of fever of unknown origin (FUO), 18 (17.3\%) of neutropenic colitis, 15 (14.4\%) of pneumonia, $13(12.5 \%)$ of mucositis, $10(9.6 \%)$ of port catheter infection, nine $(8.7 \%)$ of cellulitis, six $(5.8 \%)$ of pharyngitis, three $(2.9 \%)$ of sinusitis and one $(1.0 \%)$ case of acute ischemic stroke. Eight cases $(7.7 \%)$ had criteria for septic shock upon admission to the emergency room.

The Absolute Neutrophil Count (ANC) had a median of $193 \times 10^{3}$ (ranging from 37 to $530 \times 10^{3}$ ).

A blood culture was taken in 14 episodes (13.5\%); of these, 4 $(28.6 \%)$ were collected before the start of antimicrobial therapy and the remaining $10(71.4 \%)$ were taken afterwards.

Only one culture's result was not collected since the patient died a day after its obtaining. The remaining 13 cultures were positive; 3 $(23.1 \%)$ were gram negative bacteria and $10(76.9 \%)$ gram positive. No colonization by fungi was identified by this method. The isolated bacteria are listed in Table 3 . 
Table 3 Isolated microorganisms in positive blood cultures

\begin{tabular}{ll}
\hline Microorganism & Frequency, n (\%) \\
\hline Pseudomonas aeruginosa & $5(38.5)$ \\
Staphylococcus aureus & $4(30.7)$ \\
Klebsiella pneumoniae & $2(15.4)$ \\
Escherichia coli & $1(7.7)$ \\
Streptococcus thoralensis & $1(7.7)$
\end{tabular}

The time between the start of symptoms and the first dose of antibiotics varied from two to 36 hours and had a median of five hours. Meanwhile, time between medical contact to antibiotic administration had a median of 1.3 hours.

Beta lactams $(n=78,75.0 \%)$ and aminoglycosides $(n=72,69.2 \%)$ were the most commonly used drugs. The frequency with which these and other drugs were used can be found in Table 4.

Table 4 Antibiotic use distribution

\begin{tabular}{ll}
\hline Drug class & Frequency, n (\%) \\
\hline$\beta$-Lactams & $78(75.0)$ \\
Aminoglycosides & $72(69.2)$ \\
Vancomycin & $16(15.4)$ \\
Antifungals & $5(4.8)$ \\
Quinolones & $5(4.8)$ \\
Carbapenems & $4(3.8)$ \\
Macrolides & $3(2.9)$ \\
Trimethoprim + Sulfamethoxazole & $3(2.9)$ \\
Clindamycin & $1(1.0)$ \\
Metronidazole & $1(1.0)$
\end{tabular}

In 27 cases $(26.0 \%)$, the antimicrobial regime was not changed during the FN episode. The addition of an antifungal to antibiotic treatment occurred in seven $(6.7 \%)$ cases.

There were no complications in $78.8 \%(n=82)$ of the events. In the cases in which there were complications $(\mathrm{n}=22,31.4 \%)$, the most common was sepsis $(n=12,17.1 \%)$. The frequency of the other complications can be seen in table 5 .

Table 5 Frequency of medical complications

\begin{tabular}{ll}
\hline Complication & Frequency, n (\%) \\
\hline Acute renal injury & $5(4.8)$ \\
Hospital-acquired pneumonia & $4(3.8)$ \\
Pleural effusion & $3(2.9)$ \\
Cardiac arrest & $3(2.9)$ \\
Gastrointestinal hemorrhage & $2(1.9)$ \\
Neurological sequelae & $1(1.0)$ \\
Muscular hypotrophy & $1(1.0)$ \\
Pulmonary aspergillosis & $1(1.0)$ \\
\hline
\end{tabular}

The average hospital stay was 12 days. Twelve deaths (17.1\%) occurred as a result of an episode of FN (or its complications).

\section{Discussion}

In the study's four-year period, a total of 104 episodes of chemotherapy-associated febrile neutropenia were recorded and treated in 70 patients of GP-FMH No. 31 .

There was a marked predominance of the male gender (2.3: 1), which surpasses what was previously reported in international and national studies. ${ }^{12-14}$

The mean age was 7.6 years, higher than the study by Hakim et al. ${ }^{15}$ whose patients averaged 5.9 years, but less than the cohort of Vázquez et al., ${ }^{16}$ in Mexico, where it was 8.3 years.

The average number of events ( 1.5 per patient) was less than that found by Orozco in Mexico City children's hospital, where they registered 1.96 per child. ${ }^{17}$

Unlike the study by Düzenli et al., ${ }^{18}$ in which the majority of NF events occurred during reinduction (35.6\%), the events of the present study occurred during the induction phase (71.2\%).

FUO occupies the first cause of NF in this study, causing 27.9\% of the episodes, which coincides with other cohorts, where it explains between $23 \%$ and $53 \%$ of events. Neutropenic colitis, the second cause of FN $(17.3 \%)$, differs from these cohorts since it is only identified between 5.7 and $8.7 \%$ of the cases..$^{15,17-19}$

The median of the ANC $\left(193 \times 10^{3}\right)$ varies considerably between studies, being reported lower in some cases, as in the study of Düzenli $\left(100 \times 10^{3}\right)$, and greater in others, as in the Vanderpuye study $(200 \mathrm{x}$ $\left.10^{3}\right){ }^{18,20}$

Severe neutropenia $\left(<200 \times 10^{3}\right.$ ANC) was present in $19.3 \%$ events, lower than the study of Vathana et al., where it appeared in $44.4 \%$ laboratory findings. ${ }^{21}$

Blood culture collection, although subpar according to current treatment guidelines, ${ }^{8}$ is comparable with international and national studies, where it has varied from $9.4 \%$ to $26 \%{ }^{17,22}$ Likewise, the study by Meidani et al., showed that in $68.4 \%$ of the medical records, there was no result for culture of anybody fluid. ${ }^{23}$

Even so, blood culture results are vastly better than other studies, which have calculated rates between $0-2.6 \%$ regarding positivity, whereas $92.9 \%$ of cultures in this research were positive. ${ }^{23,24}$ The high percentage of positivity could be explained due to the fact that no evidence of negative cultures was found reported in clinical files.

Regarding microbiological isolates, the predominance of Pseudomonas aeruginosa is atypical when compared to similar studies, since it has only been positive in 5.9 to $21.6 \%$ of blood cultures. The other isolated bacteria, with the exception of S. thoralensis (which has not been identified in other series), have a comparable frequency. ${ }^{12,13,16,21}$

The time between the onset of fever and the administration of antibiotics had a median of five hours, significantly higher than what the current IDSA guideline recommends ( $<2$ hours $).{ }^{8}$ Cox et al., found that in their emergency department, $53 \%$ of patients received antibiotics during the first two hours of fever onset; only $5 \%$ of their patients took between 240-299 minutes to start treatment. ${ }^{14}$ It should be mentioned that this delay in the start of antibiotic therapy is mainly due to the parental delay to seek medical assistance. 
The time between the first medical contact and the first dose of antibiotic was 1.3 hours, which is somewhat close the current goal according to IDSA's guideline $(<1$ hour after triage). The implementation of an emergency treatment protocol, as well as adequate guidance aimed at parents could reduce the time of administration of antibiotics, as Keng et al., did in their hospital. ${ }^{25}$

The most used antibiotic regime was the combination of a $\beta$-lactam (mostly cephalosporins) with an aminoglycoside (amikacin). Similarly, in the study by Vathana et al., in Thailand, $77.1 \%$ of patients were treated with a combination of cefatazidime and gentamicin. ${ }^{21}$ In the same way, Martínez et al., found that $63 \%$ of their patients were treated with that same antibiotic combination. The additional introduction of vancomycin or antifungal therapies (6.7\%) was only necessary in a minority of patients in both studies. ${ }^{26}$

Almost a third of the patients in this study (31.4\%) presented some type of complication, lower than the study from Rodríguez et al., conducted at the Federico Gómez Children's Hospital of Mexico, whose percentage increases to $50.0 \% .^{27}$

The mean hospital stay varied considerably from patient to patient, and averaged 12 days, almost double than the study from Lal et al., who calculated an average of 7.5 days. ${ }^{28}$

\section{Conclusions}

Febrile neutropenia is a frequent complication in the pediatric population with hemato-oncological diseases in the GPH-FM No. 31. Currently, Fever of Unknown Origin is the most frequent nosological diagnosis and Gram positive bacteria are the main etiological agents. The therapeutic approach that patients receive is adequate. However, it is necessary to decrease time in antibiotic administration and to emphasize the collection of blood cultures prior to its start, in accordance with the current treatment guidelines.

\section{Acknowledgements}

None.

\section{Conflicts of interest}

The authors declare no conflict of interest.

\section{References}

1. Santolaya ME, Rabagliati R, Bidart $\mathrm{T}$, et al. Consenso de manejo racional del paciente con cáncer, neutropenia y fiebre. Rev Chil Infect. 2005;22(S2): S79-S111.

2. Hughes WT, Armstrong D, Bodey GP, et al. 2002 Guidelines for the use of antimicrobial agents in neutropenic patients with cancer. Clin Infect Dis. 2002;34(6):730-751.

3. Caggiano V, Weiss RV, Rickert TS, et al. Incidence, cost, and mortality of neutropenia hospitalization associated with chemotherapy. Cancer. 2005;103(9):1916-1924.

4. Lewis MA, Hendrickson AW, Moynihan TJ. Oncologic emergencies: Pathophysiology, presentation, diagnosis, and treatment. CA Cancer J Clin. 2011;61(5):287-314.

5. Rivas R, Best C, Fernández Y, et al. Consenso mexicano para el abordaje diagnóstico y terapéutico del paciente con neutropenia febril. Rev Hematol Mex. 2014;15(S2):S207-S268.

6. Freifeld A, Bow E, Sepkowitz K, et al. Clinical Practice Guideline for the Use of Antimicrobial Agents in Neutropenic Patients with Cancer:
2010 Update by the Infectious Diseases Society of America. Clin Infect Dis. 2011;52(4):e56-e93.

7. Alberta Health Services. Management of febrile neutropenia in adult cancer patients. 2014.

8. Lehrnbecher T, Robinson P, Fisher B, et al. Guideline for the Management of Fever and Neutropenia in Children With Cancer and Hematopoietic Stem-Cell Transplantation Recipients: 2017 Update. $J$ Clin Oncol. 2017; 35(18):2082-2094.

9. Taplitz R, Kennedy E, Bow E, et al. Outpatient Management of Fever and Neutropenia in Adults Treated for Malignancy: American Society of Clinical Oncology and Infectious Diseases Society of America Clinical Practice Guideline Update. J Clin Oncol. 2018;36(14):1443-1453.

10. Lyman GH, Kuderer NM. Filgastrim in patients with neutropenia: potential effects on quility of life. Drugs. 2002;62(Suppl 1):65-78.

11. Basu SK, Fernández ID, Fisher SG, et al. Length of stay and mortality associated with febrile neutropenia among children with cancer. J Clin Oncol. 2005;23(31):7958-7966

12. Lona J, Marín M, Cordero A, et al. Frecuencia de factores de riesgo para bacteremia en niños con cáncer, neutropenia y fiebre en un hospital de tercer nivel del occidente de México. Bol Med Hosp Infant Mex. 2013;70(4): 304-309.

13. Sano H, Kobayashi R, Iguchi A, et al. Risk factors for sepsis-related death in children and adolescents with hematologic and malignant diseases. J Microbiol Immunol Infect. 2017;50(2):232-238.

14. Cox A, Bradford N. Management of Febrile Neutropenia in Pediatric Oncology Across Queensland, Australia: A Retrospective Review on Variations Between Locations. J Pediatr Oncol Nurs. 2014;31(1):2833.

15. Hakim H, Flynn P, Knapp K, et al. Etiology and clinical course of febrile neutropenia in children with cancer. J Pediatr Hematol Oncol. 2009;31(9):623-629.

16. Vázquez J, García M, Fortanelli R, et al. Frecuencia de microorganismos aislados en pacientes pediátricos con neutropenia y fiebre que cuentan con dispositivos vasculares y su evolución clínica. Enf Inf Microbiol. 2006;26(3):66-71.

17. Orozco D. Epidemiología de los episodios de neutropenia y fiebre en los niños con Leucemia Linfoblástica Aguda en el Hospital para el Niño del Instituto Materno Infantil del Estado de México de enero del 2010 a enero del 2012. Universidad Autónoma del Estado de México. 2014, p. 39.

18. Kar YD, Özdemir ZC, Bör Ö. Evaluation of febrile neutropenic attacks of pediatric hematology-oncology patients. Turk Pediatri Ars. 2017;52(4):213-220.

19. Arnello M, Quintana J, Barraza P. Neutropenia febril en niños con cáncer en un centro asistencial en Santiago, Chile. Rev Chil Infect. 2007;24(1):27-32.

20. Vanderpuye V, Yarney J, Beecham K. Management of febrile neutropenia in patients receiving chemotherapy for solid tumors: a retrospective study of twenty cases from the radiotherapy centre, Accra, Ghana. West Afr J Med. 2010;29(5):303-308.

21. Vathana N, Thitipolpun S, Buaboonnam J, et al. Prevalence of pathogens in pediatric cancer patients with febrile neutropenia. Southeast Asian J Trop Med Public Health. 2017;48(S2):151-160.

22. Walwyn M, Nicholson A, Lee MG, et al. Febrile neutropaenia in cancer patients. West Indian Med J. 2010;59(2):209-214.

23. Meidani M, Rostami M, Moulaee S. Blood Culture in Neutropenic Patients with Fever. Int J Prev Med. 2012;3(2):141-142. 
24. Novin K, Ameri A, Yeganeh $\mathrm{SH}$, et al. Blood cultures in febrile neutropenic patients with cancer. Ann Oncol. 2016;27(S9):ix174-ix175.

25. Keng M, Thallner E, Elson P, et al. Reducing Time to Antibiotic Administration for Febrile Neutropenia in the Emergency Department. J Oncol Pract. 2015;11(6):450-455.

26. Martínez N, Vieito M, Anido U, et al. Retrospective study of febrile neutropenia. J Clin Oncol. 2017;30(15):e19505-e19505.
27. Rodríguez Y, Dorantes E, Avilés M, et al. Características clínicas y microbiológicas de eventos infecciosos en pacientes pediátricos con cáncer tratados en el Hospital Infantil de México Federico Gómez. Gac Mex Onco. 2013;12(3):150-155.

28. Lal A, Bhurgri Y, Rizvi N, et al. Factors influencing in-hospital length of stay and mortality in cancer patients suffering from febrile neutropenia. Asian Pac J Cancer Prev. 2008;9(2):303-308. 